\title{
OTIMIZAÇÃo SELETIVA COM COMPENSAÇÃO E INTERVENÇÃO GERONTOLÓGICA “LIFE-SPAN
}

\author{
Alice Bastos \\ Instituto Politécnico de Viana do Castelo - ESEVC \\ CINTESIS, AgeingC - UP \\ abastos@ese.ipvc.pt \\ Joana Monteiro \\ Instituto Politécnico de Viana do Castelo - ESEVC \\ Centro de Psicologia da Universidade do Porto - FPCEUP \\ Carla Faria \\ Instituto Politécnico de Viana do Castelo - ESEVC \\ CINTESIS, AgeingC - UP
}

Recepción Artículo: 22 mayo 2021

Admisión Evaluación: 22 mayo 2021

Informe Evaluador 1: 26 mayo 2021

Informe Evaluador 2: 27 mayo 2021

Aprobación Publicación: 01 junio 2021

\section{RESUMO}

Fatores tecnológicos, materiais, sociais e mentais associados à Cultura foram o principal motor para 0 aumento da Iongevidade no século XX e não mudanças no genoma geradas pela evolução da espécie. A otimização seletiva com compensação (SOC) pode ser vista como capacidade adaptativa - estratégias psicológicas de gestão da vida que garantem o processo de envelhecimento bem-sucedido. Inspirado nos princípios da Psicologia Desenvolvimental "Life-Span", o SOC é simultaneamente um modelo e meta-modelo, na medida em que permite explicar quer mecanismos gerais do funcionamento humano, quer transformações em domínios específicos. A Equipa de Baltes e cols. elaborou um instrumento para avaliar a capacidade adaptativa - SOCQuestionnaire, útil para a intervenção gerontológica "life-span" - uma visão otimizadora da transformação desenvolvimental ao longo da vida, tomando como referência "life-events". Este estudo analisa estratégias SOC em adultos mais velhos a participar em iniciativas autárquicas. Inclui 104 participantes, idade média 72,6 anos (DP $=5,9)$, predominantemente mulheres $(80,8 \%)$, casados $(61,5 \%)$, reduzida escolaridade $(84,6 \%)$. Na recolha de dados utilizou-se um questionário sociodemográfico e a versão portuguesa do SOC-Questionnaire. Na análise da capacidade adaptativa em função das variáveis sociodemográficas observa-se uma diferença significativa entre mulheres e homens $(p<0,05)$, assim como uma interação significativa entre grupo e anos de escolaridade ( $p<$ $0,01)$. Independentemente do grupo, as mulheres pontuam mais baixo que os homens. Nas escolaridades mais baixas, a pontuação no SOC é superior no grupo de intervenção, enquanto nas escolaridades mais altas o grupo de comparação pontua mais alto. Esta tendência nos participantes com mais escolaridade deve ser interpretada com alguma reserva, dado que há poucos participantes nestes estratos. Estes resultados parecem promissores 


\section{OTIMIZAÇÃO SELETIVA COM COMPENSAÇÃO E INTERVENÇÃO GERONTOLÓGICA “LIFE-SPAN}

para o planeamento de intervenções "life-span", enriquecendo a capacidade adaptativa ao longo da vida para lidar com situações de vida particularmente stressantes, como é o caso da Pandemia COVID-19.

Palavras chave: desenvolvimento humano; envelhecimento bem-sucedido; otimização seletiva com compensação; "SOC-Questionnaire"; intervenção gerontológica

\section{ABSTRACT}

Selective optimization with compensation and life-span gerontological intervention. Technological, material, social and mental factors associated with Culture, and not genome changes due to evoIution, were the main cause for the increase in longevity of the XX century. Selective optimization with compensation (SOC) can be considered as adaptive capacity - life management psychological strategies that lead to successful aging. Inspired in principles of Life-Span Developmental Psyhcology, SOC is simultaneously a theoretical model and a metamodel, in the sense that it explains both general mechanisms of human functioning and changes in specific domains.

Baltes and cols. created an instrument to assess adaptive capacity - SOC Questionnaire, useful to gerontological life-span intervention - an optimizing view of developmental change throughout life based on life events. The present study examines SOC strategies in older adults that take part in initiatives promoted by municipalities. There are 104 participants, with a mean age of 72,6 years $(S D=5,9)$, predominantly women $(80,8 \%)$, married $(61,5 \%)$, with reduced education (84,6\%). Data collection was performed with a sociodemographic questionnaire and the Portuguese version of the Soc Questionnaire. The analysis of adaptive capacity according to sociodemographic variables shows significant differences between women and men $(p<0,05)$, as well as a significant interaction between study group and school years $(p<0,01)$. Regardless of study group, women display lower scores than men.

Between individuals with fewer school years, SOC scores are higher in the intervention group, while among the more educated ones the comparison group has higher scores. This tendency in participants with more school years must be interpreted with caution since there are few participants in these strata. These results seem promising to plan life-span interventions, enhancing adaptive capacity throughout life in order to deal with stressful life situations, such as the COVID-10 Pandemic.

Keywords: human development; successful aging; selective optimization with compensation; "SOCQuestionnaire"; gerontological intervention

Este trabalho foi desenvolvido no âmbito de um projeto financiado pela Fundação para a Ciência e Tecnologia e Programa Operacional Competitividade e Internacionalização (SAICT-POL/23712/2016; POCI-01-0145FEDER-023712).

Os autores agradecem às Câmaras Municipais e participantes do estudo. Os agradecimentos estendem-se ainda à Professora Doutora Carolina Silva pelo apoio nas opções metodológicas e estatísticas.

\section{INTRODUÇÃO}

A Otimização Seletiva com Compensação (SOC) deve muito à visão de Paul Baltes - líder do chamado Grupo de Berlim e um dos mentores da Psicologia Desenvolvimental "Life-span". A visão que Baltes foi construindo ao longo de décadas dispõe de (1) um conjunto de princípios teóricos (Baltes, 1999, 2006), articulados com (2) um conjunto de procedimentos metodológicos - (a) instrumentos de autorrelato (ex., SOC-Questionnaire - Freund \& Baltes, 1998); (b) procedimentos de investigação altamente inovadores (ex., "think aloud" para estudar a sabedoria - Baltes \& Smith, 1990); (c) procedimentos experimentais laboratoriais, para investigar aspetos sensoriais e memória - "Testing the limits" - Baltes et al, 1989); (3) um conjunto de orientações para a intervenção psicológica/gerontológica (Baltes \& Danish, 1980). Pensando na Gerontologia - a ciência que estuda o envelhecimento, 0 velho e a velhice - a Psicologia Desenvolvimental "Life-span" fornece um quadro de referência robusto para investigar e intervir no desenvolvimento humano e envelhecimento. 
Enquanto mecanismo adaptativo, o SOC corresponde a um conjunto de estratégias psicológicas de gestão da vida que permite explicar o envelhecimento bem-sucedido (Baltes \& M. Baltes, 1990). Contrariamente à ideia amplamente divulgada, não há uma idade para entrar na velhice. Ao recusarmos a ideia da entrada numa nova fase/estádio estamos a afirmar que a ideia de envelhecimento na Psicologia e na Gerontologia precisa de ser revista para acolher a multidireccionalidade simultânea de ganhos e perdas ao longo do ciclo de vida. Mas, se pensarmos a vida dividida em três grandes períodos, observa-se que há um tempo de crescimento/ganhos (infância), um tempo de manutenção dos ganhos (meia-idade) e um tempo de gestão das perdas/declínio (velhice). Na velhice dá-se uma inversão no ratio ganhos e perdas. É aqui que o SOC ocupa um lugar central. Quanto mais Ionga a vida, maior a probabilidade de acumulo de doenças, onde se destaca a demência (Baltes \& Smith, 2003). Apesar de não haver tratamento eficaz para a demência, uma leitura de ciclo/curso de vida abre a possibilidade de prevenir este tipo de patologia (Fratiglioni et al, 2020). 0 que pode fazer a diferença no lidar com a inversão do ratio ganhos e perdas é o uso proficiente de estratégias SOC. Note-se que o modelo SOC foi apresentado no âmbito do envelhecimento bem-sucedido (Baltes \& M. Baltes, 1990), assumindo os autores que o desenvolvimento/envelhecimento é um processo que envolve três componentes - SOC - o modo como estes componentes são executados depende das circunstâncias pessoais e societais à medida que se envelhece.

No final do século XX, face à ausência de consenso relativamente a indicadores de envelhecimento bemsucedido, o modelo SOC torna-se uma das referências para investigar o processo de envelhecimento positivo. Recentemente, dados da OMS (2015) demonstram que, à medida que se avança na idade, aumenta 0 desfasamento entre saúde objetiva e subjetiva. Baltes e M. Baltes (1990) defendiam que esta situação se devia ao facto de o psiquismo humano ser muito plástico, adaptativo e capaz de compensar as perdas. Está assim dado o mote para 0 elevado potencial do SOC para a intervenção em situações patológicas (Donnellan et al, 2017; Rozario et al, 2012). Note-se que M. Baltes e Cartensen (1999) analisaram as relações entre SOC individual e SOC coletivo, lembrando que os indivíduos agem em contexto social. Portanto, o SOC pode ocorrer a nível micro (indivíduo) ou a nível macro (instituições sociais).

Nas últimas décadas, o SOC tornou-se um modelo útil para traçar planos de investigação e de intervenção sobre envelhecimento. Carpentieri et al. (2017) desenharam um estudo com o objetivo de analisar o "SOC talk", assumindo que o SOC permitia maximizar o bem-estar face ao declínio físico para executar atividades, tais como, jardinagem, reparações e cuidar da casa. Realizaram entrevistas a 15 membros de uma coorte nascida em 1936, tendo os resultados demonstrado que as pessoas que usavam estratégias SOC apresentavam valores mais altos no bem-estar, apesar da reduzida função física. Os autores concluem que o SOC pode desempenhar um papel relevante no processo de envelhecimento bem-sucedido.

Avaliar a capacidade adaptativa de modo objetivo vai ocorrer num esforço conjunto de Baltes et al. (1998, 2002). Inicialmente foi elaborado um instrumento com 42 itens, depois um de 12 itens - útil para "screening". As qualidades psicométricas mostraram-se promissoras. Todavia, tentativas de adaptação transcultural tornaram-se difíceis. Em Portugal observam-se tentativas de validação do SOC-Questionnaire (Moreira, 2019), com resultados pouco animadores. Porem, a estatística exploratória na presente amostra aponta para boa consistência interna.

Quanto à intervenção gerontológica "life-span", esta corresponde a uma visão optimizadora da transformação desenvolvimental ao longo da vida, tomando como referência "life-events"/acontecimentos de vida, em particular os não-normativos (Baltes \& Danish, 1980). A articulação entre uma visão do desenvolvimento e a intervenção é particularmente explanada neste documento publicado uma década antes do SOC. Mas as ideias estão lá, no recurso à estratégia de enriquecimento, para além da prevenção e alívio. 0 objetivo de "enriquecimento" é atingido através da otimização do funcionamento do sistema (cognitivo, comportamental, outros). Posteriormente, investigadoras de tendência desenvolvimental retomaram estas ideias (Sugarman, 2001; Viguer Segui, 2004). Assiste-se ao desenvolvimento de programas de "life skills" (Hodge et al. 2012) para aumentar reservas e lidar com "life-events". Recentemente, Paúl (2020) defende uma Gerontologia preventiva e optimizadora, mais do que "curativa" ou remediativa. 


\section{OTIMIZAÇÃO SELETIVA COM COMPENSAÇÃO E INTERVENÇÃO GERONTOLÓGICA “LIFE-SPAN}

Qual a pertinência deste estudo? Qual a relação entre SOC e iniciativas autárquicas? Se pensarmos que os "life events" desempenham um papel central na transformação desenvolvimental, então podemos pensar que otimizar o sistema individual pode ser uma forma de o tornar mais robusto. Em linha com Hodge et al. (2012), parece-nos importante aumentar os recursos internos para lidar com situações stressantes, como é o caso da Pandemia COVID-19. Assim, o esforço intencional e deliberado que alguns cidadãos fazem participando em iniciativas disponibilizadas pelas autarquias é uma forma de ir arrecadando conhecimentos, skills/capacidades e, fundamentalmente, uma atitude proativa perante a vida.

\section{OBJETIVO DE INVESTIGAÇÃO}

Analisar as estratégias SOC em adultos mais velhos que participam em projetos de envelhecimento ativo/bem-sucedido promovidos pelos municípios.

\section{PARTICIPANTES}

0 presente estudo é parte integrante de um projeto de investigação multicêntrico e multimétodo (AgeNORTC), desenvolvido em três territórios do Norte e Centro de Portugal. Neste trabalho são apresentados apenas resultados relativos a Viana do Castelo (para mais detalhes consultar Bastos et al., 2020). Trata-se de um estudo quantitativo comparativo, de natureza transversal, em que o envolvimento social é operacionalizado como participação em projetos públicos de intervenção comunitária (PPIC), implementados pelas câmaras municipais no âmbito da promoção do envelhecimento ativo/bem-sucedido. Trata-se de Iniciativas que proporcionam diversas atividades em interação com outros.

0 presente estudo incluiu 104 participantes, com idades compreendidas entre 55 e 84 anos, a viver em casa/comunidade, distribuídos em dois grupos de dimensão equivalente: (1) grupo de intervenção (GI), formado por indivíduos que frequentam PPIC ( $n=52$ ) e (2) grupo de comparação (GC), composto por indivíduos que não frequentam os PPIC ( $\mathrm{n}=52)$. $0 \mathrm{GC}$ foi emparelhado com $0 \mathrm{Gl}$ em termos de género e grupo etário.

\section{INSTRUMENTOS UTILIZADOS}

A informação foi recolhida através de um protocolo de avaliação gerontológica multidimensional composto por vários instrumentos destinados a avaliar dimensões do funcionamento individual. Para além do questionário sociodemográfico, foi utilizado o SOC-Questionnaire (Moreira, 2019). No estudo inicial as qualidades psicométricas desta medida não se mostraram muito robustas. Porém, procedeu-se a novas análises da consistência interna na presente amostra e os valores obtidos nesta amostra são bons (alpha de Cronbach - 0,73). As correlações item-total são elevadas (superiores a 0,30), com exceção de três itens (item 1, item 2, item 11). Todavia, se retirados estes itens, a consistência interna não se altera substancialmente, pelo que se manteve todos os itens.

0 processo de recolha de dados decorreu de março a maio de 2018 e toda a equipa teve formação prévia na aplicação do protocolo de recolha de dados, o qual foi administrado em locais previamente acordados. Todos os participantes foram informados acerca dos objetivos e condições de participação no estudo, tendo assinado 0 respetivo Termo de Consentimento Livre e Informado. Em termos de análises de dados, para a descrição da amostra são apresentadas as características dos participantes usando estatísticas descritivas, média e desvio padrão ou frequência. Comparámos a capacidade adaptativa no total da amostra e estratos populacionais utilizando 0 teste $t$. Na análise multivariável comparámos a capacidade adaptativa segundo as características dos participantes, incluindo na análise de covariância os fatores género, grupo, estado civil, as covariáveis idade, anos de escolaridade e rendimento (em salários mínimos) e as interações com o grupo (grupo x género, grupo x idade e grupo $x$ anos de escolaridade). Como a existência de interação com o grupo é indicativa de efeitos diferentes da intervenção em estratos populacionais, repetiu-se a análise nesses estratos. Considerou-se $p<0,05$ como indicativo de significância estatística. 


\section{RESULTADOS ALCANÇADOS}

Apresenta-se em seguida os principais resultados obtidos, designadamente caracterização dos participantes, seguida da análise da capacidade adaptativa em função das variáveis mais relevantes.

Tabela 1. Caracterização sociodemográfica da amostra

\begin{tabular}{|c|c|c|c|}
\hline \multirow[t]{2}{*}{ Características } & $\begin{array}{c}\text { Grupo de } \\
\text { Intervenção } \\
(n=52)\end{array}$ & $\begin{array}{c}\text { Grupo de } \\
\text { Comparação } \\
(n=52) \\
\end{array}$ & $\begin{array}{c}\text { Total } \\
(n=104)\end{array}$ \\
\hline & $\mathrm{n}(\%)$ & $\mathrm{n}(\%)$ & $\mathrm{n}(\%)$ \\
\hline Idade $M(D P)$ & $72,4(5,8)$ & $72,9(6,0)$ & $72,6(5,9)$ \\
\hline (Mín.-Máx.) & $61-83$ & $55-84$ & $55-84$ \\
\hline $55-74$ & $34(65,4)$ & $34(65,4)$ & $68(65,4)$ \\
\hline $75-84$ & $18(34,6)$ & $18(34,6)$ & $36(34,6)$ \\
\hline \multicolumn{4}{|l|}{ Género } \\
\hline Mulheres & $42(80,8)$ & $42(80,8)$ & $84(80,8)$ \\
\hline Homens & $10(19,2)$ & $10(19,2)$ & $20(19,2)$ \\
\hline \multicolumn{4}{|l|}{ Estado Civil } \\
\hline Solteiro & $2(3,8)$ & $3(5,8)$ & $5(4,8)$ \\
\hline Casado(a)/União de Facto & $32(61,5)$ & $32(61,5)$ & $64(61,5)$ \\
\hline Separado(a)/Divorciado(a) & $5(9,6)$ & $1(1,9)$ & $6(5,8)$ \\
\hline Viúvo(a) & $13(25,0)$ & $16(30,8)$ & $29(27,9)$ \\
\hline \multicolumn{4}{|l|}{ Escolaridade } \\
\hline Sem escolaridade & $0(0,0)$ & $4(7,7)$ & $4(3,8)$ \\
\hline $1^{\circ}-4^{\circ}$ Anos & $46(88,5)$ & $38(73,1)$ & $84(80,8)$ \\
\hline $5^{\circ}-6^{\circ}$ Anos & $1(1,9)$ & $1(1,9)$ & $2(1,9)$ \\
\hline $7^{\circ}-9^{o}$ Anos & $2(3,8)$ & $3(5,8)$ & $5(4,8)$ \\
\hline $10-12^{\circ}$ Anos & $3(5,8)$ & $5(9,6)$ & $8(7,7)$ \\
\hline Ensino Superior & $0(0,0)$ & $1(1,9)$ & $1(1,0)$ \\
\hline \multicolumn{4}{|l|}{ Rendimento Mensal $^{\mathrm{a}}$} \\
\hline Reduzido $(\leq 750 €)$ & $25(49,0)$ & $25(50,0)$ & $50(49,5)$ \\
\hline Médio/Elevado $(>750 €)$ & $26(51,0)$ & $25(50,0)$ & $51(50,5)$ \\
\hline Vive com Outras Pessoas & $37(71,2)$ & $40(76,9)$ & $77(74,0)$ \\
\hline
\end{tabular}

Nota. ${ }^{\text {a }}$ Registou-se um valor omisso no grupo de comparação.

Como se observa na Tabela 1, os projetos públicos de intervenção comunitária (PPIC) são frequentados predominantemente por mulheres, da terceira idade, com escolaridade reduzida e a residir com outras pessoas maioritariamente com o cônjuge. 


\section{OTIMIZAÇÃO SELETIVA COM COMPENSAÇÃO E INTERVENÇÃO GERONTOLÓGICA “LIFE-SPAN}

Tabela 2. Capacidade adaptativa (média e desvio padrão) em função das variáveis sociodemográficas

\begin{tabular}{|c|c|c|c|c|}
\hline Variáveis & $\begin{array}{c}\text { Grupo de } \\
\text { Intervenção }\end{array}$ & $\begin{array}{c}\text { Grupo de } \\
\text { comparação }\end{array}$ & Teste $t$ & $\begin{array}{c}\text { Valor de } \\
p\end{array}$ \\
\hline Todos & $6,7(2,8)$ & $6,3(2,9)$ & 0,7 & 0,5 \\
\hline $\begin{array}{l}\text { Idade } \\
\begin{array}{r}55-74 \\
75-84\end{array}\end{array}$ & $\begin{array}{l}6,5(2,8) \\
7,1(2,8)\end{array}$ & $\begin{array}{l}6,7(3,0) \\
5,4(2,6)\end{array}$ & $\begin{array}{l}0,3 \\
1,8\end{array}$ & $\begin{array}{c}0,7 \\
0,08\end{array}$ \\
\hline $\begin{array}{l}\text { Género } \\
\text { Feminino } \\
\text { Masculino }\end{array}$ & $\begin{array}{l}6,4(2,9) \\
7,8(2,5)\end{array}$ & $\begin{array}{l}6,1(2,8) \\
7,2(3,2)\end{array}$ & $\begin{array}{l}0,6 \\
0,5\end{array}$ & $\begin{array}{l}0,6 \\
0,6\end{array}$ \\
\hline $\begin{array}{l}\text { Estado civil } \\
\text { Casado } \\
\text { Outros }\end{array}$ & $\begin{array}{l}6,6(2,2) \\
6,9(3,7)\end{array}$ & $\begin{array}{l}6,8(3,2) \\
5,5(2,2)\end{array}$ & $\begin{array}{l}0,3 \\
1,4\end{array}$ & $\begin{array}{l}0,7 \\
0,2\end{array}$ \\
\hline $\begin{array}{l}\text { Escolaridade } \\
<4 \text { anos } \\
4 \text { anos } \\
5-9 \text { anos } \\
10 \text { ou mais anos }\end{array}$ & $\begin{array}{l}6,1(2,5) \\
7,3(2,7) \\
5,7(3,7) \\
3,0\end{array}$ & $\begin{array}{l}5,4(2,3) \\
5,9(2,9) \\
9,0(1,4) \\
8,4(3,7)\end{array}$ & $\begin{array}{l}0,7 \\
1,8 \\
1,9 \\
1,3\end{array}$ & $\begin{array}{c}0.5 \\
0,08 \\
0,09 \\
0,3\end{array}$ \\
\hline $\begin{array}{l}\text { Rendimento } \\
\text { Reduzido }(\leq 750 €) \\
\text { Médio/Elevado }(>750 €)\end{array}$ & $\begin{array}{l}6,2(2,7) \\
6,7(2,9)\end{array}$ & $\begin{array}{l}6,4(2,8) \\
6,2(3,0)\end{array}$ & $\begin{array}{l}0,3 \\
0,6\end{array}$ & $\begin{array}{l}0,7 \\
0,5\end{array}$ \\
\hline
\end{tabular}

Na análise das estratégias SOC em função das variáveis sociodemográficas (Anexo - Tabela 1) existe uma diferença significativa $(F(1,94)=4,9 ; p<0,05)$ entre mulheres $(M=6,3 ; D P=2,8)$ e homens $(M=7,5 ; D P=2,8)$ e uma interação significativa entre 0 grupo e anos de escolaridade $(F(1,94)=8,4 ; p<0,01)$. Independentemente do grupo, as mulheres pontuam sempre mais baixo que os homens e, como pode observar-se na Tabela 2, nas escolaridades mais baixas ( $<4$ e 4 anos) a pontuação no SOC é superior no grupo de intervenção, enquanto que nas mais altas (5-9 e $\geq 10) 0$ grupo de comparação pontua mais alto (Figura 1). Esta tendência nos participantes com mais escolaridade deve ser interpretada com alguma reserva, dado que há poucos participantes nestes estratos (ver Tabela 1), e apenas um participante no grupo de intervenção tem 10 anos de escolaridade e os 5 participantes no grupo de comparação têm 11, 12 e 17 anos de escolaridade. 
Figura 1. Capacidade adaptativa (média e intervalo de confiança a 95\%) segundo a escolaridade nos dois grupos

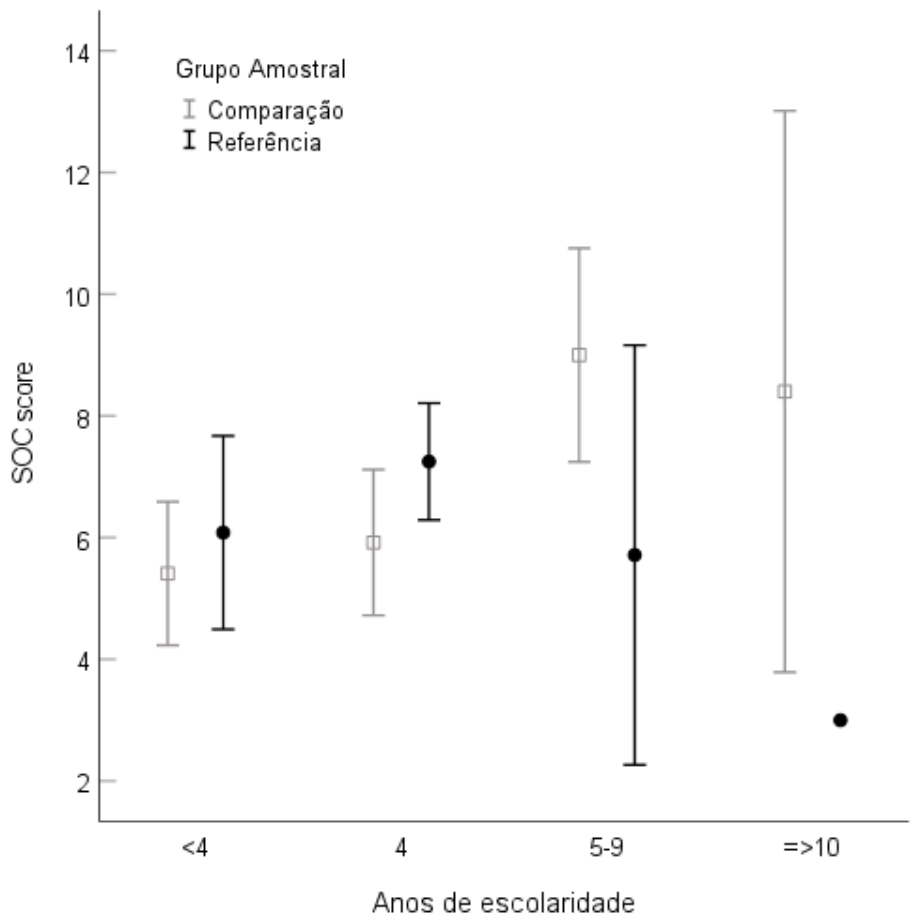

Procederíamos seguidamente com uma análise mais detalhada nos grupos com baixa escolaridade $(\leq 4$ anos, $n=86)$ e os restantes ( $n=18)$, mas atendendo à reduzida dimensão amostral neste último grupo, analisámos apenas o grupo menos escolarizado. Repetindo a análise de covariância (Anexo - Tabela 2) vemos que existe uma diferença significativa $(F(1,82)=5,6 ; p<0,05)$ entre os grupos de intervenção $(M=6,9 ; D P=2,6)$ e comparação $(M=5,7 ; D P=2,7)$ e permanece a diferença significativa $(F(1,82)=6,0 ; p<0,05)$ entre mulheres $(M=$ $6,0 ; \mathrm{DP}=2,6)$ e homens $(M=7,7 ; \mathrm{DP}=2,9)$. 


\section{OTIMIZAÇÃO SELETIVA COM COMPENSAÇÃO E INTERVENÇÃO GERONTOLÓGICA “LIFE-SPAN}

Figura 2. Capacidade adaptativa (média e intervalo de confiança a 95\%) nos participantes com escolaridade $\leq$ 4 anos $(n=86)$ segundo o grupo etário e género nos 2 grupos
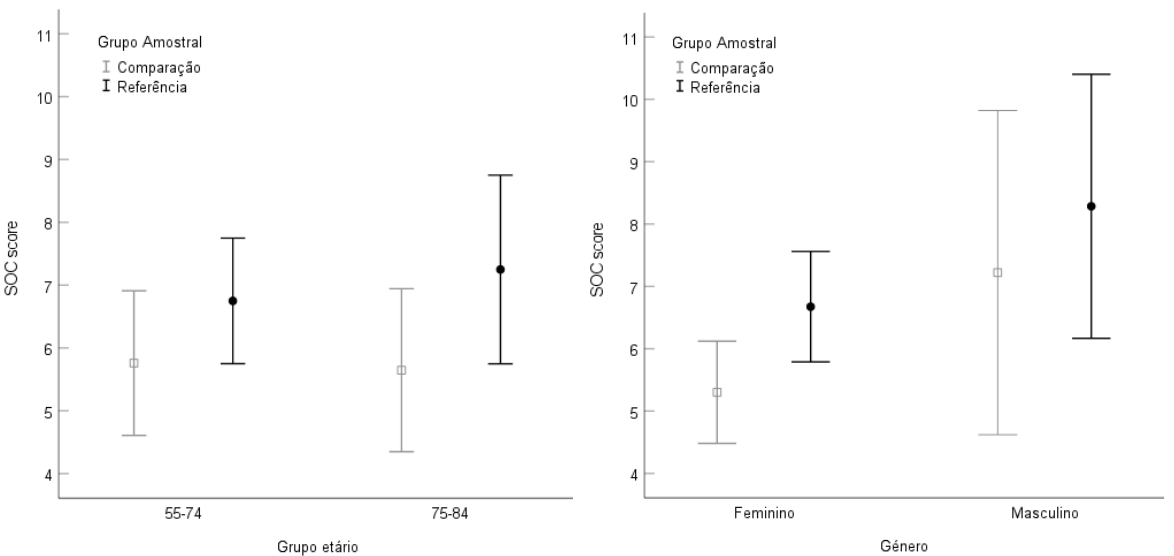

As interações entre grupo e género ou idade não são significativas (Figura 2), indicando que a intervenção tem um efeito positivo nas estratégias SOC em homens e mulheres e não dependente da idade.

\section{DISCUSSÃO}

Face ao objetivo estabelecido, no presente estudo observa-se que os projetos públicos de intervenção comunitária são frequentados predominantemente por mulheres, da terceira idade, com escolaridade reduzida e a residir com outras pessoas - maioritariamente com o cônjuge. Na análise das estratégias SOC (i.e., capacidade adaptativa) em função das variáveis sociodemográficas observa-se uma diferença significativa entre mulheres e homens, assim como uma interação significativa entre 0 grupo e anos de escolaridade. Independentemente do grupo, as mulheres pontuam mais baixo que os homens e nas escolaridades mais baixas a pontuação no SOC é superior no grupo de intervenção, enquanto nas escolaridades mais altas o grupo de comparação pontua mais alto. Esta tendência nos participantes com mais escolaridade deve ser interpretada com alguma reserva, dado que há poucos participantes nestes estratos. Face a estes resultados, analisámos apenas o grupo menos escolarizado repetindo a análise de covariância. Observou-se uma diferença significativa entre os grupos de intervenção e comparação e permaneceu a diferença significativa entre mulheres e homens. As interações entre grupo e género ou idade não foram significativas, indicando que a intervenção tem um efeito positivo sobre as estratégias SOC em homens e mulheres e independentemente da idade.

Para melhor se compreender os resultados obtidos, parece-nos relevante retomar aqui algumas das ideias centrais da capacidade adaptativa, visto que estes mecanismos SOC correspondem à operacionalização do instrumento usado neste estudo. 0 mecanismo de seleção refere-se à restrição nos domínios de funcionamento do indivíduo porque há perda no potencial adaptativo (P. Baltes \& M. Baltes, 1990). A tarefa adaptativa é concentrarse então em domínios de prioridade elevada e envolve a convergência entre exigências ambientais e motivações, skills e capacidades individuais. Embora a seleção esteja conotada com a redução do número de domínios de elevada eficácia, pode também envolver novos e transformados domínios e objetivos de vida. Posteriormente, a seleção vai ser desdobrada entre seleção eletiva (representa as escolhas possíveis dentro de um conjunto de alternativas), e seleção baseada na perda como consequência das oportunidades limitadas e perdas associada à "Iate life" (Baltes et al, 1999, 2006). Este desdobramento é indicativo das mudanças profundas que ocorrem ao longo do ciclo de vida. 
Por seu turno, a otimização reflete a visão de que as pessoas se envolvem em comportamentos para enriquecer e aumentar as suas reservas gerais e maximizar escolhas em termos de quantidade e qualidade (Baltes \& M. Baltes, 1990). Investigação sobre plasticidade demonstra que as pessoas mais velhas continuam a ser capazes de executar este processo de otimização (consultar investigação associada ao procedimento "testing the limits" e "memorizing while walking" - Lindenberger et al., 2000; Kliegl et al, 1989). Refere-se à aplicação de métodos ou procedimentos para conseguir atingir os objetivos selecionados. Este processo pode envolver a aquisição de procedimentos (aprender um novo "skill"), ou ainda usar meios em comportamentos orientados para objetivos relevantes (por ex., aplicando o skill). 0 processo de otimização do comportamento pode também ser considerado no quadro de regulação do comportamento. Um dos exemplos que ilustra a relevância da otimização é 0 treino-prática, situação que pode aproximar-nos da perfeição apesar da incompletude da arquitetura humana (Baltes, 1997).

Finalmente, a compensação envolve aspetos da mente e da tecnologia, resulta de uma restrição no leque de plasticidade (leia-se potencial adaptativo), tal como a seleção; torna-se operativa quando se perde capacidades comportamentais específicas ou as capacidades se reduzem abaixo do padrão exigido para funcionamento adequado (Baltes \& M. Baltes, 1990). Refere-se ao uso de métodos alternativos, quando métodos anteriormente usados não estão disponíveis. A compensação distingue-se da seleção na medida em que a seleção se refere à escoIha de objetivos mais do que a escolha de métodos. Ao contrário da otimização, a compensação define-se pela substituição de métodos - ou seja, à medida que um conjunto de métodos se torna menos usado, outro conjunto 0 vai substituir. Daí a importância da mente (ex., uso de mnemónicas) e da tecnologia (ex., dispositivos face à redução da visão, audição, outros) à medida que a idade avança, em particular na $4^{a}$ idade (85+ anos) onde 0 ratio entre ganhos e perdas é cada vez menos favorável (Lindenberger, 2014).

Face ao exposto, 0 que há de importante a afirmar sobre o presente estudo? Observa-se que há características pessoais que parecem ser diferenciadoras na capacidade adaptativa e que 0 envolvimento em atividades sociais pode contribuir para aumentar o potencial adaptativo à medida que a longevidade avança. Todavia, importa ter presente a especificidade dos mecanismos SOC e o valor global do SOC-Questionnaire.

Face às limitações deste estudo, recomenda-se prosseguir com o estudo reavaliando os mesmos participantes no pós-pandemia COVID-19, uma vez que se trata de um acontecimento de vida com potencial impacto na capacidade adaptativa. Igualmente, recomenda-se aprofundar o estudo da capacidade adaptativa, designadamente através do "SOC talk", para melhor ajustar os planos de intervenção às características das pessoas.

\section{CONCLUSÕES}

A revolução da longevidade colocou novos desafios à Humanidade. 0 envelhecimento humano é um processo multifacetado, regulado por múltiplas influências (normativas e não normativas), onde as capacidades individuais e os dispositivos da Cultura ocupam um lugar de destaque. Disponibilizar iniciativas/projetos às populações para manter tanto quanto possível o contacto social, é um dos maiores desafios que se coloca às Políticas Públicas. A pandemia COVID-19 provavelmente vai transformar-se num dos "life-events" relevantes do seculo XXI, com impacto na vida coletiva (acontecimento histórico) e também em algumas vidas em particular (acontecimento não-normativo), pelas perdas sociais, doença grave, outros.

Considerando os achados obtidos neste estudo, há algumas variáveis que parecem contribuir significativamente para a capacidade adaptativa, nomeadamente características associadas ao género e à escolaridade. Em termos da intervenção gerontológica "Life-span", o SOC-Questionnaire parece-nos um instrumento de trabalho relevante para planear e avaliar a eficácia das intervenções preventivas e optimizadoras. Porém, sendo esta uma amostra com poucos participantes em algumas das categorias de análise, estes resultados promissores devem ser vistos com alguma cautela. 


\section{OTIMIZAÇÃO SELETIVA COM COMPENSAÇÃO E INTERVENÇÃO GERONTOLÓGICA “LIFE-SPAN}

\section{REFERÊNCIAS BIBLIOGRÁFICAS}

Baltes, M. \& Carstensen, L. (1999). Social-psychological theories and their applications to aging: From individual to collective. In V. L. Bengston, \& K. W. Schaie, Handbook of Theories of Aging (209-226). Springer.

Baltes, P. (1987). Theoretical propositions of life-span developmental Psychology: On the dynamics between growth and decline. Developmental Psychology, 23(5), 611-626. 10.1037/0012-1649.23.5.611

Baltes, P. \& Baltes, M. (1990). Psychological perspectives on successful aging: The model of selective optimization with compensation. In P. Baltes \& M. Baltes (Eds.), Successful aging: Perspectives from the behavioural sciences (pp. 1-34). Cambridge University Press.

Baltes, P. \& Danish, S. (1980). Intervention in life-span development and aging. In R. Turner \& H. Reese (Eds), Life-span developmental psychology - Intervention (pp. 49-78). Academic Press.

Baltes, P., Lindenberger, U. \& Staudinger, U. (2006). Life span theory in developmental psychology. In R. Lerner \& W. Damon (Eds.), Handbook of child psychology: Theoretical models of human development (pp. 569664). John Wiley.

Baltes, P. \& Smith, J. (2003). New frontiers in the future of aging: From successful aging of young old to the dilemmas of the fourth age. The Gerontologist, 49(2), 123-135. 10.1159/000067946

Baltes, P., Staudinger, U. \& Lindenberger, U. (1999). Lifespan Psychology: Theory and application to intellectual functioning. Annual Review of Psychology, 50(1), 471-507.

Bastos, A., Monteiro, J., Faria, C., Pimentel, H., Silva, S., \& Afonso, C. (2020). Participação em programas de intervenção comunitária e qualidade de vida: Resultados de um estudo multicêntrico em Portugal. Revista Brasileira de Geriatria e Gerontologia, 23(6), Artigo e19007. 10.1590/1981-22562020023.190017

Carpentieri, J., Elliott, J., Brett, C. \& Deary, I. (2017). Adapting to aging: Older people talk about their use of selection, optimization, and compensation to maximize well-being in the context of physical decline. The Journals of Gerontology Series B: Psychological Sciences and Social Sciences, 72(2), 351-361, 10.1093/geronb/gbw132.

Donellan, C., Hevey, D., Hickey, A. \& O'Neil, D. (2012). Adaptation to stroke using a model of successful aging. Aging, Neuropsychology, and Cognition, 19(4), 530-547. 10.1080/13825585.2011.638976

Fratiglioni, L., Marseglia, A., \& Dekhtyar, S. (2020). Ageing without dementia: Can stimulating psychosocial and lifestyle experiences make a difference? The Lancet, 9(6), 533-543, 10.1016/S1474-4422(20)30039-9.

Freund, A., \& Baltes, P. (1998). Selection, optimization, and compensation as strategies of life management: Correlations with subjective indicators of successful aging. Psychology and Aging, 13(4), 531543. 10.1037/0882-7974.13.4.531

Freund, A. \& Baltes, P. (2002). Life-management strategies of selection, optimization and compensation: Measurement by self-report and construct validity. Journal of Personality and Social Psychology, 82(4), 642662. 10.1037/0022-3514.82.4.642

Hodge, K., Danish, S. \& Martin, J. (2012). Developing a conceptual framework for life skills interventions. The Counseling Psychologist, 41(8), 1125-1152, 10.1177/0011000012462073.

Kliegl, R., Smith, J., \& Baltes, P. (1989). Testing-the-limits and the study of adult age differences in cognitive plasticity of a mnemonic skill. Developmental Psychology, 25(2), 247-256. 10.1037/0012-1649.25.2.247

Lindenberger, U. (2014). Human cognitive aging: corriger la fortune? Science, 346(6209), 572-578. 10.1126/science. 1254403

Lindenberger, U., Marsiske, M., \& Baltes, P. B. (2000). Memorizing while walking: Increase in dual-task costs from young adulthood to old age. Psychology and Aging, 15(3), 417-436. 10.1037/0882-7974.15.3.417

Moreira, B. (2019). A Otimização Seletiva com Compensação no processo de envelhecimento: Um estudo preliminar com o "SOC-Questionnaire" IPVC-ESE. http://hdl.handle.net/20.500.11960/2219

Organização Mundial de Saúde (2015). World report on ageing and health. http://apps.who.int/iris/bitstream/handle/10665/186463/9789240694811_eng.pdf?sequence=1 
Paúl, C. (2020). Prefácio. In R. Benavente (Coord.), Intervençao psicológica em gerontologia (pp. XIII-XVI). Pactor.

Rozario, P., Kidahashi, M., DeRienzis, D. (2011). Selection, Optimization and compensation: Strategies to maintain, maximize, and generate resources in later life in face of chronic illnesses. Journal of Gerontological Social Work, 54(2), 224-239. 10.1080/01634372.2010.539589

Smith, J., \& Baltes, P. (1990). Wisdom-related knowledge: Age/cohort differences in response to life-planning problems. Developmental Psychology, 26(3), 494-505. 10.1037/0012-1649.26.3.494

Sugarman, L. (2001). Life-span development. Frameworks, accounts and strategies. Routledge.

Viguer Segui, P. (2004). Optimizacion evolutiva: Fundamentos del desarrollo optimo. Ediciones Piramide.

\section{Anexo}

ANEXO - Tabela 1

Análise de covariância do SOC score

\begin{tabular}{l|r|r|r|r|r} 
Source & $\begin{array}{c}\text { Sum of } \\
\text { Squares }\end{array}$ & df & Mean Square & \multicolumn{1}{c}{ F } & \multicolumn{1}{c}{ Sig. } \\
\hline Corrected Model & $132,859^{\mathrm{a}}$ & 9 & 14,762 & 1,974 &, 051 \\
\hline Intercept & 49,576 & 1 & 49,576 & 6,628 &, 012 \\
\hline Grupo &, 235 & 1 &, 235 &, 031 &, 860 \\
\hline Sexo & 36,920 & 1 & 36,920 & 4,936 &, 029 \\
\hline Idade & 3,379 & 1 & 3,379 &, 452 &, 503 \\
\hline AnosEscola & 3,269 & 1 & 3,269 &, 437 &, 510 \\
\hline Rendimento & 5,820 & 1 & 5,820 &, 778 &, 380 \\
\hline casado &, 513 & 1 &, 513 &, 069 &, 794 \\
\hline Grupo * Sexo & 1,131 & 1 & 1,131 &, 151 &, 698 \\
\hline Grupo * Idade & 1,325 & 1 & 1,325 &, 177 &, 675 \\
\hline Grupo * AnosEscola & 62,626 & 1 & 62,626 & 8,372 &, 005 \\
\hline Error & 703,131 & 94 & 7,480 & & \\
\hline Total & 5217,000 & 104 & & & \\
\hline Corrected Total & 835,990 & 103 & & & \\
\hline a. R Squal & & & & &
\end{tabular}

a. R Squared $=, 159$ (Adjusted R Squared $=, 078$ )

ANEXO - Tabela 2

Análise de covariância do SOC score (Escolaridade $<=4$ anos)

\begin{tabular}{l|r|r|r|r|r} 
Source & $\begin{array}{c}\text { Sum of } \\
\text { Squares }\end{array}$ & df & Mean Square & \multicolumn{1}{c|}{ F } & \multicolumn{1}{c}{ Sig. } \\
\hline Corrected Model & $73,385^{\mathrm{a}}$ & 3 & 24,462 & 3,661 &, 016 \\
\hline Intercept & 18,052 & 1 & 18,052 & 2,702 &, 104 \\
\hline Grupo & 37,520 & 1 & 37,520 & 5,616 &, 020 \\
\hline Sexo & 40,288 & 1 & 40,288 & 6,030 &, 016 \\
\hline Idade &, 533 & 1 &, 533 &, 080 &, 778 \\
\hline Error & 547,836 & 82 & 6,681 & & \\
\hline Total & 4075,000 & 86 & & & \\
\hline Corrected Total & 621,221 & 85 & & & \\
\hline
\end{tabular}

a. R Squared $=, 118$ (Adjusted R Squared $=, 086)$ 
